\title{
Comparison of quantification from field deployable pXRF and laboratory based- micro-XRF within an SEM of Cu-based alloys
}

\author{
Thomas Lam
}

Smithsonian Institution, Museum Conservation Institute, Suitland, Maryland, United States

With the non-destructive nature of portable X-ray fluorescence ( $\mathrm{pXRF}$ ) spectrometry and the development of laboratory SEM-based micro-XRF systems, this study aims to compare the quantification results of the two techniques [1]. Being that quantification of $\mathrm{Cu}$ based alloys XRF have been well published and is an area of interest in cultural heritage since the late 1950s, six Cu based alloys National Bureau of Standards (NBS), now National Institute of Standards and Technology (NIST), standard reference materials (SRM) were selected for this study that have certified chemistries [2]. The six NBS SRMs were SRM 1102, SRM 1104, SRM 1105, SRM 1107, SRM 1110, and SRM 1114 [3,4]. Though SRM 1102, SRM 1104, and SRM 1105 are now archived, the certified chemistries are still easily obtainable [4]. The composition range of these SRMs allowed for the experimental assessment of $\mathrm{Cu}$ in the concentration range of 61.183 to 96.45 mass\%, and $\mathrm{Zn}$ in the concentration range 3.47 to 37.396 mass\%. For pXRF, a Bruker Elio with a Rh source that collimates to forms an X-ray spot $\approx 1 \mathrm{~mm}$ was operated at $50 \mathrm{kV}$ and 20 micro-amps. X-ray collection was performed in air for a time of 240 seconds. For micro-XRF, a Bruker XTrace (performed in vacuum in a Hitachi $3700 \mathrm{~N}$ scanning electron microscope (SEM)) using a Rh source with a polycapillary optics to form an X-ray spot of $\approx 33$ micron $(\mathrm{Cu} \mathrm{K})$, was operated at $50 \mathrm{kV}$ and 600 microamps. A Bruker 6|60 SDD was used in the collection of X-rays for 120 live seconds. Three measurements were performed for each sample for both $\mathrm{pXRF}$ and micro-XRF. For quantification, $\mathrm{pXRF}$ fundamental parameters (FP) approach was computed by the Bruker Elio 1.6.0.42 software and micro-XRF FP approach was computed using Bruker Esprit 2.1 [1]. Table I are the quantified results for the six NBS SRM Cu-based alloys in comparison with the certified actual values. Slightly larger standard deviation was observed for the micro-XRF measurements due to the microanalytical nature which is not unexpected, as SRMs are not microbeam standards. Figures 1A-D, are the computed FP quant from both pXRF and micro-XRF compared to the actual concentrations for $\mathrm{Cu}, \mathrm{Zn}, \mathrm{Sn}$, and $\mathrm{Pb}$. The data for $\mathrm{Cu}$ (Figure 1A) and Zn (Figure 1B) from both techniques are in linear agreement with the 1 to 1 line for concentration computed by FP quant compared to the actual concentration, providing a confidence in the bulk measurement. A difference in the detectability limit for Sn (Figure 1C) between pXRF and micro-XRF was observed. The computed FP quant was less than actual certified value of $\mathrm{Pb}$ for both techniques (Figure 1D). Interestingly, through comparison of the two techniques, this study also found that pXRF does appear to have sensitivity for Fe to as low as 0.043 mass\% and $\mathrm{Ni}$ as low as 0.07 mass \%; however, the computed FP quant is an over calculation. In pXRF an observed systematic Fe and Ni baseline signal at these low concentrations suggest the $\mathrm{Fe}$ and $\mathrm{Ni}$ are artifacts from the detector. The study will discuss these in greater detail. 
Table I. Cu based alloys measured from pXRF and micro-XRF compared with the actual value (in mass\%).

\begin{tabular}{c|c|c|c|c|c|c|c}
\multicolumn{2}{c}{} & SRM 1102 & SRM 1104 & SRM 1105 & SRM 1107 & SRM 1110 & SRM 1114 \\
\hline \multirow{3}{*}{$\mathrm{Cu}$} & p-XRF & $72.72 \pm 0.04$ & $61.75 \pm 0.21$ & $64.22 \pm 0.09$ & $61.38 \pm 0.04$ & $84.24 \pm 0.02$ & $95.81 \pm 0.02$ \\
& micro-XRF & $71.81 \pm 0.17$ & $61.84 \pm 0.72$ & $64.19 \pm 0.39$ & $61.30 \pm 0.21$ & $84.75 \pm 0.08$ & $96.37 \pm 0.03$ \\
& Actual & 72.85 & 61.33 & 63.72 & 61.183 & 84.59 & 96.45 \\
\hline \multirow{3}{*}{$\mathrm{Zn}$} & p-XRF & $27.01 \pm 0.03$ & $35.17 \pm 0.13$ & $33.81 \pm 0.11$ & $36.93 \pm 0.01$ & $15.44 \pm 0.01$ & $3.95 \pm 0.02$ \\
& micro-XRF & $27.18 \pm 0.17$ & $35.53 \pm 0.09$ & $34.05 \pm 0.29$ & $37.3 \pm 0.22$ & $15.19 \pm 0.08$ & $3.53 \pm 0.04$ \\
& Actual & 27.10 & 35.31 & 34.03 & 37.396 & 15.20 & 3.47 \\
\hline \multirow{3}{*}{$\mathrm{Sn}$} & p-XRF & n.d. & $0.47 \pm 0.02$ & $0.25 \pm 0.01$ & $1.19 \pm 0.01$ & n.d. & n.d. \\
& micro-XRF & n.d. & $0.38 \pm 0.04$ & $0.20 \pm 0.04$ & $1.17 \pm 0.26$ & n.d. & $0.05 \pm 0.04$ \\
& Actual & 0.006 & 0.43 & 0.20 & 1.066 & 0.019 & 0.027 \\
\hline \multirow{4}{*}{$\mathrm{Pb}$} & Elio & n.d. & $1.87 \pm 0.08$ & $1.36 \pm 0.04$ & $0.13 \pm 0.01$ & n.d. & n.d. \\
& Xtrace & n.d. & $2.10 \pm 0.66$ & $1.47 \pm 0.14$ & $0.13 \pm 0.02$ & n.d. & n.d. \\
& Actual & 0.02 & 2.77 & 2.01 & 0.185 & 0.033 & 0.012 \\
\hline \multirow{3}{*}{$\mathrm{Fe}$} & p-XRF & $0.10 \pm 0.02$ & $0.14 \pm 0.02$ & $0.13 \pm 0.02$ & $0.09 \pm 0.01$ & $0.11 \pm 0.03$ & $0.09 \pm 0.02$ \\
& micro-XRF & n.d. & $0.09 \pm 0.01$ & $0.06 \pm 0.01$ & $0.04 \pm 0.01$ & $0.04 \pm 0.00$ & $0.04 \pm 0.01$ \\
& Actual & 0.011 & 0.088 & 0.043 & 0.0389 & 0.033 & 0.017 \\
\hline \multirow{3}{*}{$\mathrm{Ni}$} & p-XRF & $0.18 \pm 0.01$ & $0.29 \pm 0.05$ & $0.22 \pm 0.01$ & $0.29 \pm 0.02$ & $0.21 \pm 0.01$ & $0.15 \pm 0.01$ \\
& micro-XRF & n.d. & $0.04 \pm 0.00$ & $0.02 \pm 0.00$ & $0.07 \pm 0.00$ & $0.02 \pm 0.00$ & $0.02 \pm 0.00$ \\
& Actual & 0.005 & 0.07 & 0.043 & 0.0946 & 0.053 & 0.021
\end{tabular}
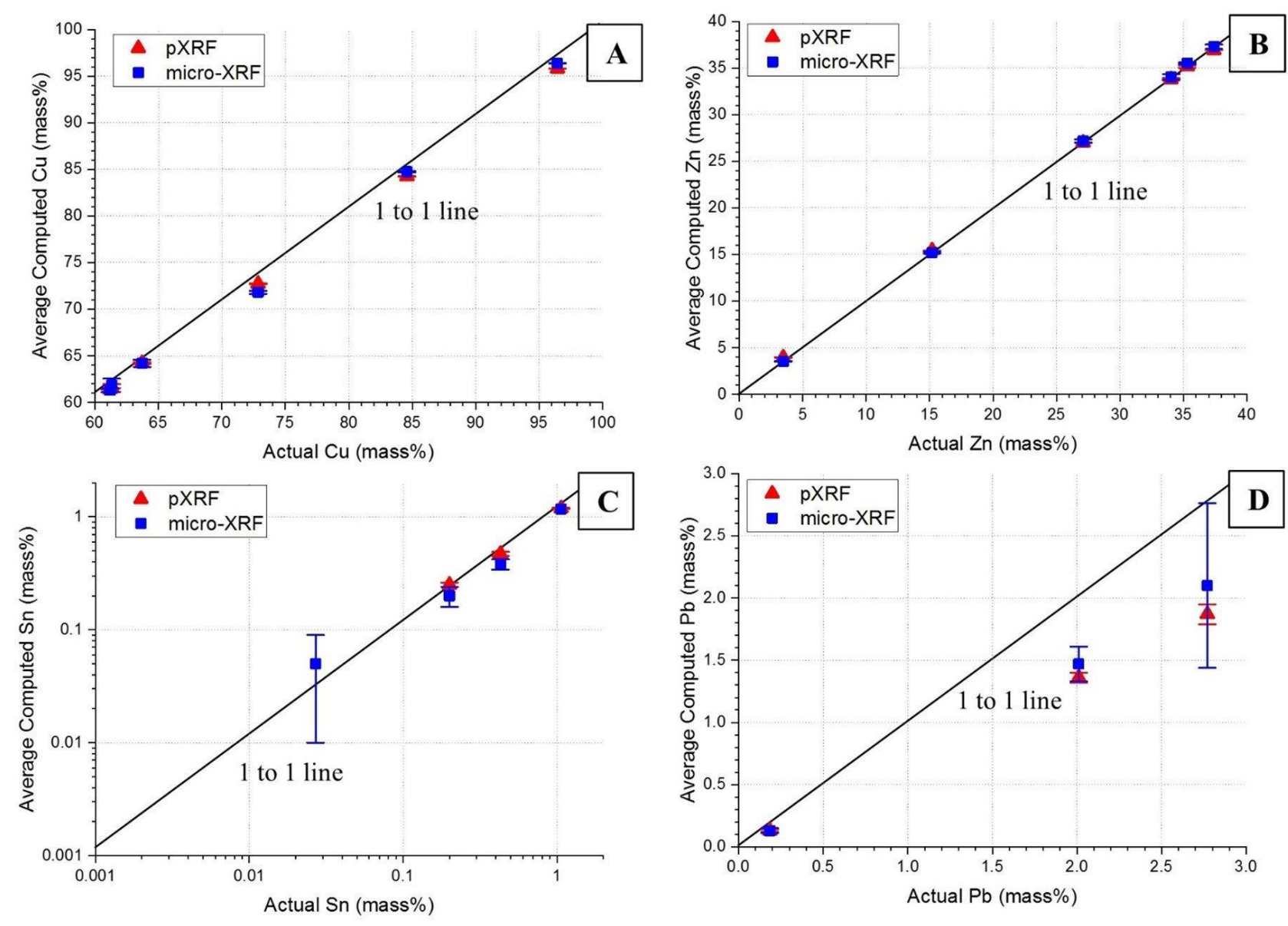
Figure 1. Computed FP quant from both $\mathrm{pXRF}$ and micro-XRF (y-axes) compared to the actual concentration (x-axes) for (A) $\mathrm{Cu}(\mathrm{B}) \mathrm{Zn}(\mathrm{C}) \mathrm{Sn}(\mathrm{D})$ and $\mathrm{Pb}$.

\section{References}

[1] M. Haschke, Laboratory Micro-X-Ray Fluorescence Spectroscopy: Instrumentation and Applications. Springer (2014).

[2] A. Heginbotham et al., Archaeometry, 57, 5 (2015) 856-868.

[3] https://www-s.nist.gov/srmors/detail.cfm?searchstring=metal\&Tables=GO

[4] https://www-s.nist.gov/srmors/certArchive.cfm 